\title{
Problematização dos MOOC na atualidade: Potencialidades e Desafios
}

\author{
Cláudia Smaniotto Barin - Centro de Ciências Naturais e Exatas - UFSM - \\ claudiabarin@nte.ufsm.br
}

Fábio da Purificação de Bastos - Centro de Educação, Departamento de Metodologia do Ensino - UFSM - fabio@ufsm.br

\begin{abstract}
Resumo
O objetivo deste artigo é avaliar o desenvolvimento dos cursos abertos massivo online (MOOC), suas potencialidades, desafios e perspectivas na atualidade. O estudo consiste de uma revisão bibliográfica. Realizou-se, para isso, a mineração de trabalhos científicos sobre o assunto no Portal de Periódicos CAPES utilizando como palavraschave "MOOC" e "Massive Open Online Courses", priorizando-se artigos avaliados por pares e publicados nos últimos cinco anos (2008-2013). Após criteriosa análise foram selecionados 16 artigos que possuíam como descritores os termos: "perspectives", "advances" ou "challenges". Os dados obtidos apontam para as potencialidades dos MOOC como forma de socialização do aprendizado de qualidade e com baixo custo, oportunizando a formação e capacitação da população, alterando os espaços de ensinar e aprender, requerendo em contrapartida uma nova postura das instituições de ensino e seus profissionais.
\end{abstract}

Palavras-chave: cursos aberto massivos online; aprendizagem em rede; tecnologias da informação e comunicação; inovação disruptiva

\begin{abstract}
This aim of this paper is to evaluate the development of massive online open courses (MOOC), and its potential, challenges and prospects today. The study consists of the bibliographic research and analysis of origins, structure and trajectories of MOOC. Held, for this, the mining of scientific papers on the subject in Journal Portal CAPES using as keywords "MOOC" and "Massive Open Online Courses", prioritizing peerreviewed articles published in the last four years (2009-2013). After careful analysis we selected 16 articles that had the terms as descriptors: "perspectives", "advances" or "challenges". The data indicate the potential of the MOOC as a form of socialization of learning quality and cost, providing opportunities for training and education of the population, changing spaces of teaching and learning, requiring in turn a new position of educational institutions and their professionals.

Keywords: massive open online courses; net learning; information and communications technology; disruptive innovation
\end{abstract}

\section{Introdução}

Os cursos abertos online e massivos (MOOC) são cursos ofertados mediante as ferramentas da web 2.0, em ambientes virtuais de ensino-aprendizagem (AVEA) ou 
redes sociais e visam oferecer para um grande número de alunos a oportunidade de ampliar seus conhecimentos num processo de co-produção.

Motta e Inamorato (2012) apontam duas características essenciais dos MOOC: são cursos abertos, no entanto requerem dos estudantes "letramento digital" e permitem escalabilidade, ou seja, a arquitetura do curso permite atender ao aumento exponencial de inscrições, podendo chegar a centenas de milhares de estudantes participando em cada oferta de curso.

Os MOOC têm se disseminado por meio de diversas plataformas espalhadas pelo mundo, tais como o Coursera, disponível no endereço https://www.coursera.org, o Udacity (http://www.udacity.com) e o OpenClass (http://www.openclass.com), atraindo milhares de adeptos. O que diferencia um MOOC de uma iniciativa tradicional de EaD é principalmente sua abrangência, visto que são planejados para atender a um número elevado de estudantes, além de ser em sua maioria gratuitos e de curta duração.

A Coursera, empresa americana que fez parceria com algumas das melhores universidades do mundo, ultrapassou novas fronteiras da educação online, ofertando a partir de 2012, quarenta e três cursos nas áreas de saúde, tecnologia, ciências humanas e astronomia. O lançamento oficial da organização foi em abril. Poucas semanas depois, a Coursera já contava com cerca de 700 mil alunos participando dos chamados MOOC (HOUGLAND, 2012).

De acordo com Izumi (2013), o Brasil é o segundo país em número de inscritos em cursos online gratuitos oferecidos por universidades estrangeiras pelo site Coursera. Dos cerca de 2 milhões de alunos, 5,9\% são brasileiros; só atrás dos norte-americanos (38,5\%). Na sequência aparecem Índia (5,2\%) e China (4,1\%). Aqui no Brasil o modelo teve seu início com o projeto MOOC EaD, que organiza a história da $\mathrm{EaD}$ no Brasil utilizando os recursos de timeline do Facebook.

Neste sentido, o presente trabalho apresenta uma problematização dos MOOC na atualidade: potencialidades (resultados alcançados; relatos de experts; possibilidades acadêmico/pedagógicas); desafios (problemas detectados; relatos de experts; críticas acadêmico/pedagógicas) e oportunidades que estão sendo vislumbradas (perspectivas).

\section{Metodologia:}

O presente trabalho consiste em um estudo descritivo e bibliográfico desenvolvido a partir de artigos científicos sobre o tema estudado, ou seja, num processo de levantamento e análise da produção bibliográfica, fornecendo uma visão geral e evidenciando novas idéias a respeito dos MOOC. Realizou-se, para isso, a mineração de trabalhos científicos sobre o assunto no Portal de Periódicos CAPES utilizando como palavras-chave "MOOC” e "Massive Open Online Courses".

Deu-se prioridade aos artigos avaliados por pares e publicados nos últimos cinco anos (2008-2013), escritas em português, inglês ou espanhol e que apresentaram textos completos disponíveis. Após criteriosa análise foram selecionados 16 artigos que possuíam como descritores os termos: “perspectives”, “advances” ou "challenges” que serviram de base para elaboração deste trabalho.

\section{Desenvolvimento}

Os resultados provenientes das buscas pelas palavras-chave apontam como principais tópicos relacionados à publicação: o ensino superior, a internet e a educação online, sendo relevante o número de artigos na área de tecnologias educacionais e cursos online, conforme pode ser visualizado na Figura 1. 

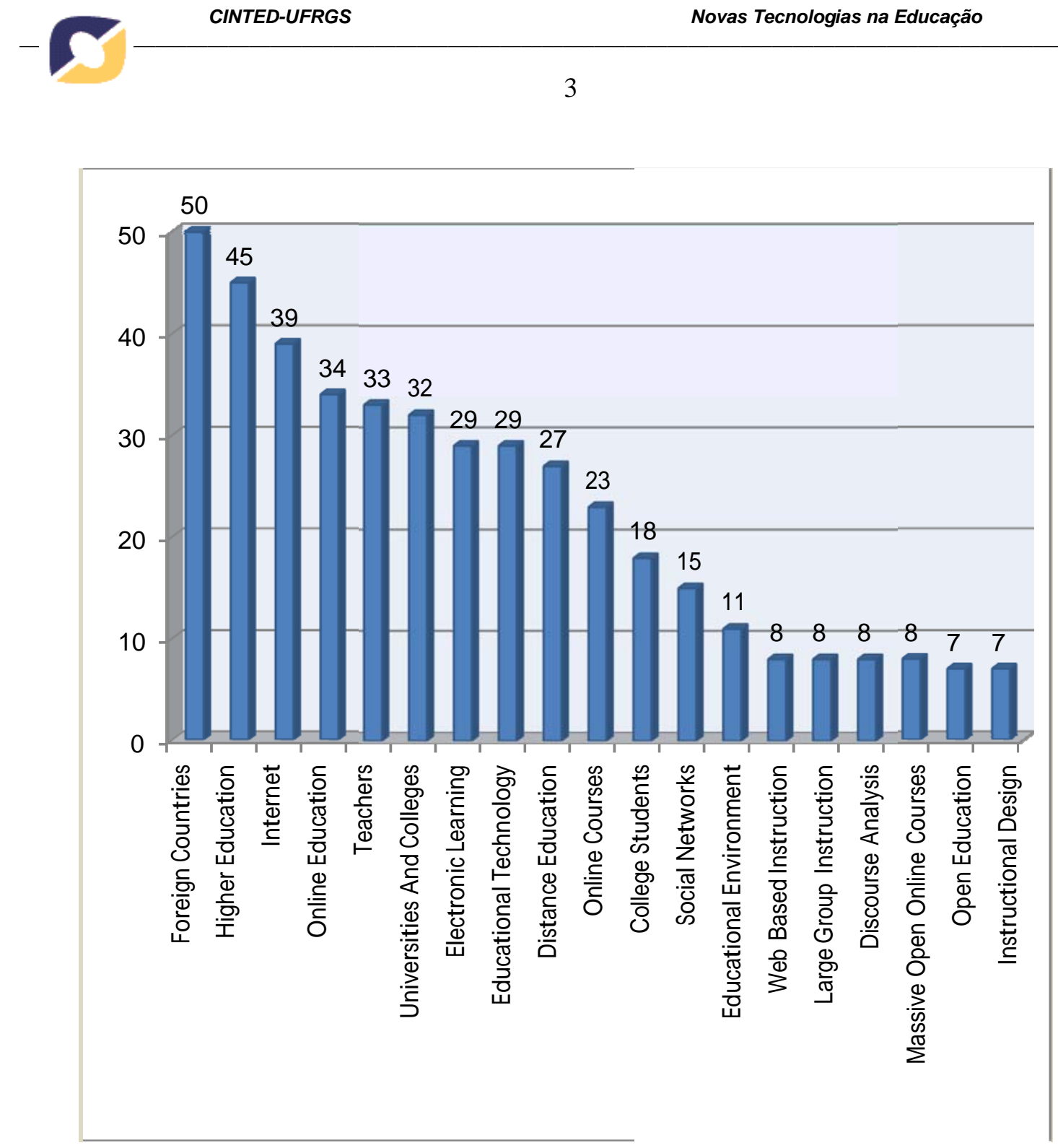

Figura 1 - Correlação entre número de artigos e tópicos descrita no Portal de Periódicos CAPES.

Em relação à análise sobre as principais bases de dados onde os artigos encontram-se disponibilizados pode-se observar (Fig.2) que a maior parte dos trabalhos encontram-se nas bases GALE e Web of Science sendo seguidas da Medline, SpringLink Open Acess e Elsevier (Science Direct).

As pesquisas e relatos descritos nos 16 artigos selecionados embasam as problematizações a deste trabalho. 


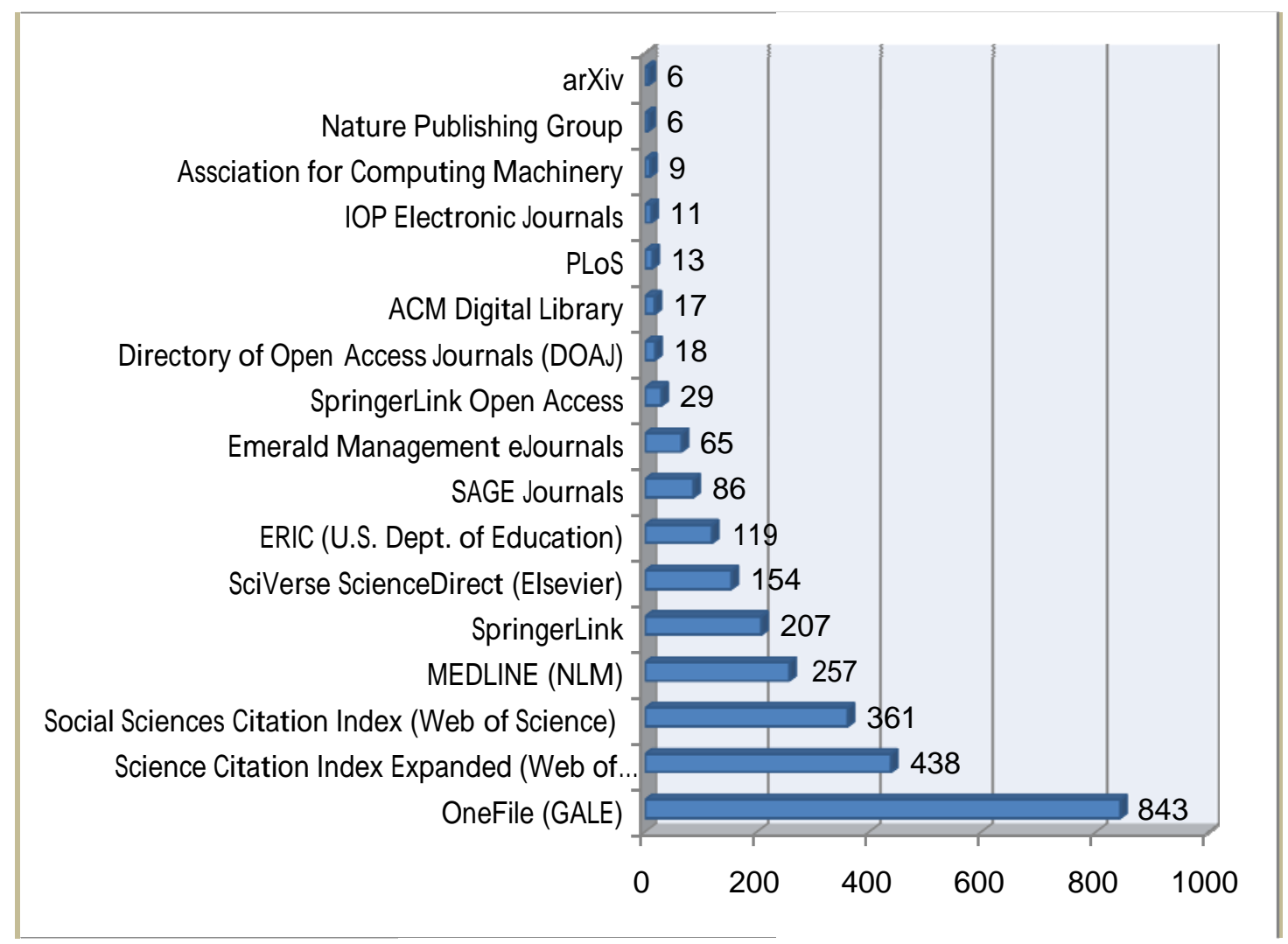

Figura 2 - Correlação entre número de artigos e bases de dados descrita no Portal de Periódicos CAPES.

\section{Potencialidades}

Little (2013) relata em seu artigo que o termo MOOC foi cunhado por George Siemens e Stephen Downes, os instrutores do primeiro curso on-line: "conectivismo e conhecimento conjuntivo (CCK08)" um curso oferecido online e em tempo real pela Universidade de Manitoba, no outono de 2008 (apud Mahraj, 2012). A inscrição foi aberto a qualquer pessoa com uma conexão à Internet, sem nenhum custo, mas os alunos que queriam computar os créditos do curso necessitavam se registrar e pagar taxa de matrícula. Em 2010 e 2011 MOOCs ganharam maior destaque depois do professor da Stanford, Sebastian Thrun e o diretor de pesquisa da Google, Peter Norvig, ofertaram o curso "CS221" sobre inteligência artificial. Enquanto palestras online ou gravações de aulas são estáticos e passivos e proporcionam baixa interatividade aos estudantes, com pouco mais do que uma lista de leitura e Slides do PowerPoint, um MOOC está estruturado para ser muito mais interativo e é esse o segredo do sucesso.

Hyman (2012) avalia o ano de 2012 como o ano em que as TIC popularizam a educação por meio dos MOOC ou cursos abertos massivos on-line, que perturbaram ou transformaram as formas de ensinar e aprender. De acordo com o autor as universidades norte-americanas, como Stanford, MIT, Harvard, Princeton, Universidade de Michigan, e a Universidade da Pensilvânia facilitaram o acesso ao ensino superior na medida em que disponibilizaram algumas de suas salas de aula online. Uma das mais bem sucedidas disciplinas on-line, foi a "Introdução à Inteligência Artificial" ministrado por Peter Norvig, diretor do Google de pesquisa, e Sebastian Thrun, 
vice-presidente da Google, no ano passado. Mais de 160.000 estudantes se inscreveram e 23.000 concluíram o curso.

Alisson et al. (2012) afirmam que a Web tem possibilitado alternativas criativas para a transformação dos espaços de ensinar e aprender por meio das TIC. No entanto, o autor ressalta que apesar de ter ocorrido muitas inovações e sucessos de significância no contexto educacional, houve também na última década muitos objetivos não atingidos. De acordo com os autores, o uso da web 2.0 trouxe consigo uma grande teia de questionamentos sobre aprendizagem formal e informal, formatação de materiais para o ensino mediado por tecnologias, gestão da aprendizagem materiais e processos em AVEA, potencialidades e desafios da integração de recursos interativos, entre outros. Além disto, o autor sugere que novos desafios devam surgir com o surgimento dos MOOC e da Web 3D imersiva para a próxima geração de AVEA.

Martin (2012) afirma que a universidade moderna é um ecosssitema muito maior que seu elenco de cursos. O autor afirma que grande parte do entusiasmo de trabalhar com os MOOC advém da potencialidade de ofertar uma educação de qualidade com baixo custo, além de fazer parte de uma grande comunidade de aprendizado, onde educandos e educadores fazem parte do processo.

\footnotetext{
"Na melhor das hipóteses, podemos realmente agregar valor, por ser professores. Podemos depurar individualmente os pensamento dos estudantes, orientá-los em projeto e, honestamente, ser entusiasta quando eles se superam” (MARTIN, 2012).
}

A oferta de MOOC levantam questões legais e políticas significativas para biblioteca de recursos educacionais, que muitas vezes são feitas para apoiar o desenvolvimento destes cursos. Essas questões envolvem preocupações políticas de informação e autoria que são centrais para as bibliotecas de recursos educacionais, incluindo a correta aplicação do "fair use", a transição para o acesso aberto como o modo padrão de publicação acadêmica, e a prestação de igualdade de acesso à aprendizagem com materiais para alunos com e sem deficiência (Butler, 2012)

Desafios

Anderson (2005) citado por Fini (2012), afirma que o papel das comunidades que utilizam software social educacional como um fator atenuante chave contra o isolamento de alunos de ritmo próprio de assimilar e internalizar o conhecimento, em ambientes virtuais de ensino-aprendizagem.

Demillo (2012) ressalta que para que as TIC transformem o modelo de aprendizagem e efetivamente enraizem suas vantagens faz-se necessário uma maior engajamento da comunidade científica, citando como exemplo o surgimento do quadronegro em sala de aula que teve sucesso imediato devido ao baixo custo, alta usabilidade e necessidade desprezível de manutenção, sendo apontado pelo autor como a última invenção que teve valor pedagógico tão óbvio, que se tornou um elemento onipresente em sala de aula.

Para Fini (2009) com o advento dos MOOC, surge a necessidade de pesquisar questões sobre o perfil dos estudantes, especialmente no que se refere aos resultados e impactos do curso, além de avaliar de forma mais profunda questões acerca da sustentabilidade e carga de trabalho dos instrutores a fim de compreender de forma mais afetiva o custo e a eficácia destas iniciativas.

Skiba (2012) aponta que os MOOC são uma inovação disruptiva no ensino superior e como tal apresentam opiniões positivas e negativas na blogosfera. O autor relata que os cursos abertos têm apresentado grande crescimento com a criação das companhias como o Corsera, EdX e Udacity. Outro aspecto a ser considerado é que 
universidades como a do estado do Colorado (EUA) tem computado três créditos para estudantes que concluem o curso de Introdução a Ciência da Computação em cursos abertos online.

Milmann (2012) aponta que apesar dos MOOC apresentarem alta taxa de matrículas, a permanência dos estudantes em cursos não pagos é pequena, sendo este fator, segundo a autora, um dos maiores desafios dos cursos massivos online gratuitos.

Alguns dos principais desafios e paradigmas a serem enfrentados na oferta de cursos do tipo MOOC:

- Como e se será fornecida a certificação/validação cursos o que implica diretamente no número de interessados

- Professores precisam também aprender a lidar com os novos papéis em uma rede de ambientes de aprendizagem online

- Muitos cursos possuem licença aberta de seus conteúdos sendo permitido reuso ou customização de materiais, entretanto outros MOOC têm baseado seus recursos educacionais com proteção de direitos autorais.

- Nem todos os cursos abertos são gratuitos, algumas instituições cobram taxas de estudantes que desejam ganhar créditos universitários e é provavel que isto se torne cada vez mais comum;

Clarke (2013), afirma ainda que dentre os desafios do surgimento dos MOOC no ensino superior encontram-se:

- Possibilidade de criação de seu próprio MOOC, embora segundo o autor isto requeira a mobilização de recursos e construção de consórcios eficazes;

- Adesão a um consórcio MOOC existente, embora os grupos sejam bastante fechados;

- Garantir que os MOOC e os cursos ofertados na Universidade possuem o mesmo padrão de qualidade de conteúdo e sofisticação tecnológica;

Assim pode-se afirmar que os cursos abertos do tipo MOOC, possibilitam uma alternativa para dois dos três desafios da educação de nível superior: acesso e custo. Programas baseados em MOOC não só possibilitam a democratização à educação, mas também a sua escalabilidade poderia contribuir de forma afinca para findar a trajetória insustentável de aula.

\section{Oportunidades}

Fini (2009) relata que com o surgimento dos MOOC no cenário do e-learning, o aprendizado contínuo ganha várias ferramentas para contrução e gestão de redes de aprendizagem, além de oferecer oportunidades para testar tais redes. Neste sentido, os MOOC, possibilitam a investigação de estudantes ao longo da vida e suas atidudes em relação a aprendizagem em rede, tanto no que se refere a flexibilidade do aprendizado, a gestão do conhecimento, usabilidade e potencialidades das ferramentas de aprendizagem baseadas na web, que proporcionam interação e interatividade e requerem a autonomia do aprendizado.

O autor ainda aponta que seus estudos tem demonstrado que o nível de reflexão sobre o uso das tecnologias em rede para o aprendizado é elevado e que os cursos abertos online estimulam a integração de ferramentas da web, assim combinações do Moodle, blogs e wiki potencializam a aprendizagem de estudantes (FINI, 2009). 
Johnson et.al (2012) afirmam que a educação constitui de elemento fundamental para o progresso da sociedade e para o bem estar social e econômico. Por outro lado, os autores relatam o fato da educação em todos os níveis ser cara e muitas vezes ineficaz. Neste sentido surgem, portanto novos paradigmas: Como educar com menor custo, com qualidade e de forma mais ágil e eficiente? Os cursos abertos online apontam como tendência de inovação para o ensino, com menor custo e maior flexibilidade atendendo assim as demandas crescentes da sociedade.

Segundo Tschoffen e Mackness (2012) a conectividade desponta como teoria alternativa de aprendizado na era digital que se baseia em quatro princípios: conectividade, liberdade (flexibilidade de tempo e espaço), diversidade (plataformas de aprendizagem, recursos educacionais e atividades de estudo) e autonomia. O campo exploratório destes princípios tem sido os MOOC, que propiciam uma larga fonte de dados para estudos de tutoria inteligente, sistemas de mineração de dados, psicologia da aprendizagem em ambientes interativos, ecologias cognitivas, etc.

Os cursos massivos online além de envolverem um elevado número de estudantes possibilitam acomodar uma vasta gama de níveis de participação e envolvimento do estudante devido sua programação assíncrona e flexível, possibilitando ao estudante um aprendizado dinâmico, com oportunidade de "engajamento" social e colaborativo de aprendizagem. Estes cursos surgem como grande oportunidade de aprendizagem ao longo da vida (SKIBA, 2012).

Alisson 2012 a comunicação on-line do grupo, simulações, laboratórios virtuais, multimídia ricos, a entrada no mundo real, a integração da aprendizagem formal e informal e apoio para adaptação e desenvolvimento através da análise on-line do uso indivíduos fazem do aprendizado interativo recursos.

Demillo (2012) afirma que as faculdades e universidades americanas vivem um colapso que tem sido retratado em inúmeros trabalhos. No entanto, no meio deste cenário de dúvidas surgem inúmeros avanços nas TIC proporcionando uma nova concepção de ensino, reduzindo custos, ampliando o acesso, melhorando resultados e aumentando transparência financeira.

De transformação cultural, do sistema educacional e, por conseguinte da sociedade os MOOC possuem como elemento invariável as plataformas online de ensino, todos eles são oferecidos no formato online, entretanto alguns possuem também um componente presencial. Os MOOC vieram pra ficar e contribuíram no desenvolvimento de ambientes adaptativos e avaliações automatizadas. Além disto, estes cursos possibilitam um novo conceito de negócios que poderão gerar receita às instituições. No entanto o autor relata a necessidade futura de propiciar diferentes caminhos para a participação dos estudantes minimizando assim a alta taxa de evasão que tem sido observada (FASIMPAUR, 2013).

Anderson e McGreal (2012) apontam que o acrescimento do custo da educação superior resulta na inacessibilidade de milhões de aprendizes em potencial e sugerem que a cursos abertos online podem prover uma redução nos custos do ensino superior com qualidade similar aos cursos presenciais tradicionais. De acordo com os autores afirmam que as características disruptivas dos MOOC surgem como oportunidade de prover inovações no ensino superior.

Com base nos autores citados podemos afirmar que os MOOC surgem como alternativa viável para a democratização do conhecimento num processo de co-autoria e co-produção utilizando ferramentas da web 2.0. Esta modalidade de curso permite a flexibilização do ensino no que se refere ao tempo e a potencialidade dos recursos educacionais hipermidiáticos. A figura 3 apresenta um mapa conceitual contendo os principais desafios, potencialidades e oportunidade dos MOOC na atualidade. 


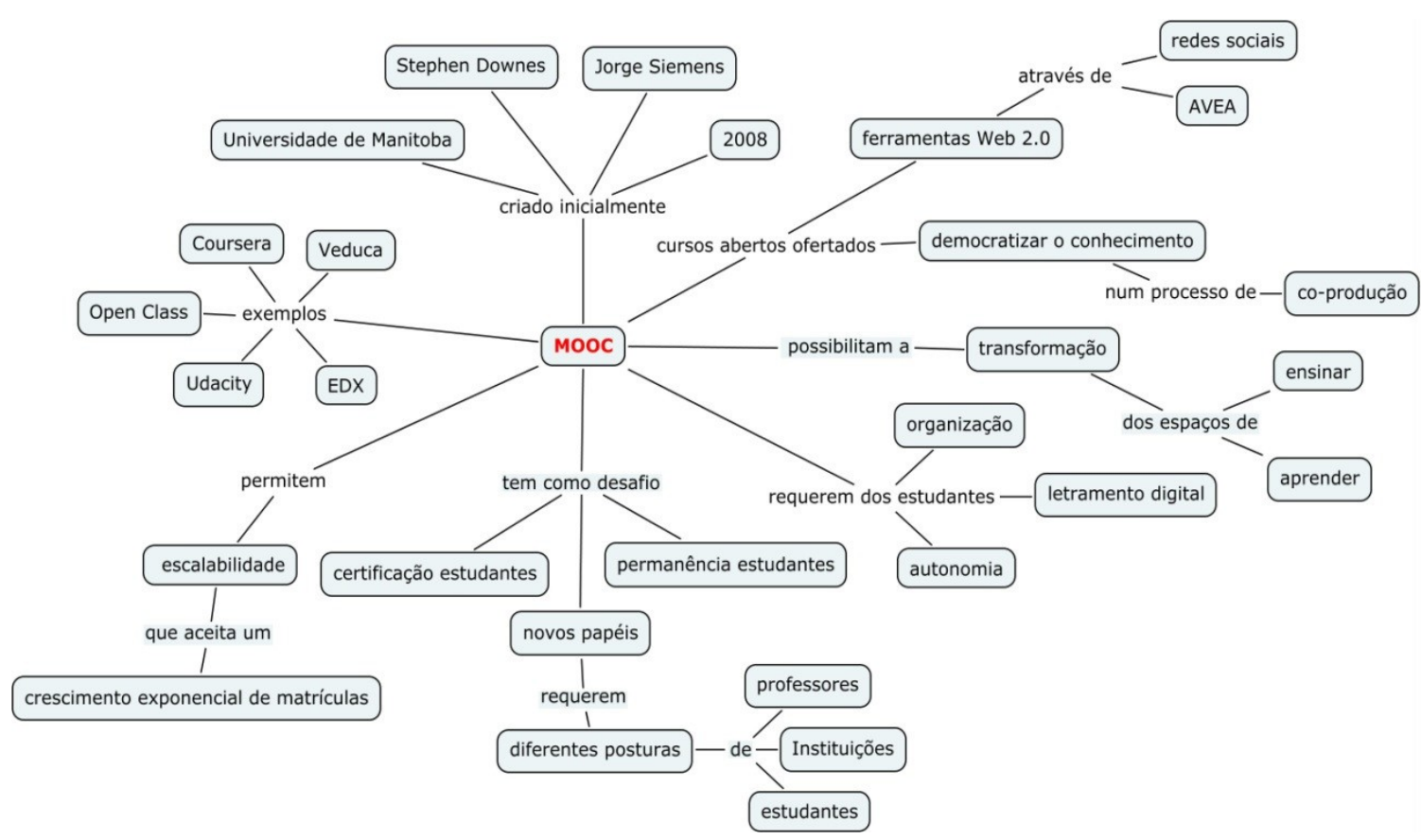

Figura 3 - MOOC desafios e potencialidades

Como se pode observar, o sucesso da aprendizagem nestes cursos depende não só do planejamento e estratégias de ensino propostas, mas sim do letramento digital, organização e autonomia dos estudantes, assim como de uma nova postura das instituições de ensino e dos professores.

\section{Considerações Finais}

A inovação tecnológica já é uma realidade em diversos ramos da sociedade como na indústria e no comércio. Assim, pode-se afirmar que a chegada das tecnologias influencia o modus operandi da sociedade e consequentemente requer uma nova visão sobre os atuais paradigmas educacionais.

Nesta perspectiva os cursos abertos massivos surgem como uma oportunidade de formação e capacitação da população, alterando os espaços de ensinar e aprender e requerendo assim uma nova postura das instituições de ensino e seus profissionais.

Os cursos abertos massivos online em virtude de seu elevado número de estudantes apresentam-se como campo de pesquisa potencial para o estudo e criação de recursos e atividades de ensino mais flexíveis e interativos, bem como de novas estratégias de avaliação.

No entanto, ainda é preciso empreender mais esforços para investigar de que forma o aprendizado interativo aberto proporcionará mudanças nos processos e práticas de ensino. A estimativa é de que a melhoria da infraestrutura interligada no acréscimo do entendimento do que é educacionalmente eficaz resultará em avanços sobre o uso da Web para a aprendizagem ao longo da próxima década.

\section{Agradecimento: Apoio CAPES}




\section{Bibliografia}

ALLISON, C.; MILLER, A.; OLIVER, I.; MICHAELSON, R.; TIROPANIS, T.. The Web in education. Computer Networks, v.56, p. 3811-3824, 2012.

ANDERSON, T.. Distance learning - social software’s killer app? (2005).

Disponível em:

http://www.unisa.edu.au/odlaaconference/PPDF2s/13\%20odlaa\%20-\%20Anderson.pdf.

Acesso em junho de 2013.

ANDERSON, T.; McGREAL, R.. Disruptive Pedagogies and Technologies in Universities. Educational Technology and Society, v.15, n.4, p. 380-389, 2012.

BUTLER, B .. Massive Open Online Courses: Legal and Policy Issues for Research Libraries. Association of Research Library. Disponível em: http://www.arl.org/ bm doc/issuebrief-mooc-22oct12.pdf.

CLARKE, T.. The advance of the MOOCs (massive open online courses) The impending globalization of business education? Education + Training, v.55, n.4-5, p.403-413,2013.

COOPER, S.; SAHAMI, M. Education Reflections on Stanford's MOOCs: New possibilities in online education create new challenges. Communications of the ACM, v.56, n.2, p.28-30, 2013.

DEMILLO, R.A.. Keeping Technology Promises: Considering new models for educational technology and methods Communications of the ACM v. 55, n.11, p. 3739, 2012.

IZUMI, C.E. Brasil é o segundo país em número de inscritos em cursos online de universidades americanas. Disponível em: http://educacao.uol.com.br/noticias/2013/01/28/brasil-e-osegundo-pais-em-numero-de-inscritos-em-cursos-online-do-coursera.htm . Acesso em maio de 2013.

FASIMPAUR, K.. Massive and Open. Learning \& Leading with Technology. v.40, n.6, p.12-17, 2013.

FINI, A.. The Technological Dimension of a Massive Open Online Course: The Case of the CCK08 Course Tools. International Review of Research in Open and Distance Learning, v.10, n. 5, p. 1-26, 2009.

FLYNN, J.T.. Moocs: disruptive innovation and the future of higher education. Christian Education Journal. v.10, n.1, p. 149-162, 2013.

HOUGLAND, L.. Universidades americanas oferecem mais de 100 cursos online gratuitos. BBC Brasil. Disponível em: http://www.bbc.co.uk/portuguese/noticias/2012/07/120719_cursos_online_lh.shtml. Acesso em maio de 2013. 
HYMAN, P.. In the Year of Disruptive Education: as college tuitions soar, various online models vie to educate college students worldwide-at no cost. Communications of the ACM, v.55, n. 12, p. 20-22, 2012

JOHNSON, J.; BUCKINGHAM SHUM, S.; WILLIS, A.; BISHOP, S.; ZAMENOPOULOS, T.; SWITHENBY, S.; MACKAY, R.; MERALI, Y.; LORINCZ, A.; COSTEA, C.; BOURGINE, P.; LOUÇÃ, J.; KAPENIEKS, A.; KELLEY, P.; CAIRD, S.; BROMLEY, J.; DEAKIN CRICK, R.; GOLDSPINK, C.; COLLET, P.; CARBONE, A.; HELBING, D.. The FuturICT education accelerator. Eur. Phys. J. Special Topics. v.214, p.215-243, 2012.

LITTLE, G.. Managing Technology: Massively Open? The Journal of Academic Librarianship, v.39, p. 308-309, 2013.

MARTIN, F.G.. Will Massive Open Online Courses Change How We Teach? Communications of the ACM , v.55, n. 8, p.26-28, 2012.

MILMAN, N.B.. MOOCs What are day? Plus 20 Questions We Should Be Asked About Them. Distance Learning, v.9, n.4, p.91-93, 2012.

MOTTA, R.; INAMORATO, A.. MOOC, uma revolução em curso. Jornal da Ciência, novembro de 2012. Disponível em:

http://www.jornaldaciencia.org.br/Detalhe.jsp?id=85111. Acesso em junho de 2013.

SKIBA, D. J.. Disruption in Higher Education: Massively Open Online Courses (MOOCs). Nursing Education Perspectives, v. 33, n. 6, p. 416-417, 2012.

TSCHOFEN, C. \& MACKNESS, J. Connectivism and dimensions of individual experience. The International Review of Research in Open and Distance Learning, v.13, n.1, p.124-143, 2012. Disponível em:

http://www.irrodl.org/index.php/irrodl/article/view/1143/2086 . Acesso em maio de 2013. 